\title{
X-ray activity and rotation of the young stars in IC 348
}

\author{
F. Alexander and T. Preibisch
}

\author{
Universitäts-Sternwarte München, Ludwig-Maximilians-Universität, Scheinerstr. 1, 81679 München, Germany \\ e-mail: [frauke; preibisch]@usm. uni-muenchen.de
}

Received 15 September 2011 / Accepted 15 December 2011

\section{ABSTRACT}

\begin{abstract}
Context. The physical origin of the strong magnetic activity in T Tauri stars and its relation to stellar rotation is not yet wellunderstood.

Aims. We investigate the relation between the X-ray activity, rotation, and Rossby number for a sample of young stars in the $\approx 3 \mathrm{Myr}$ old cluster IC 348.

Methods. We use the data of four Chandra observations of IC 348 to derive the X-ray luminosities of the young stars. Basic stellar parameters and rotation rates are collected from the literature. This results in a sample of $82 \mathrm{X}$-ray detected stars with known rotation periods. We determine the Rossby numbers (i.e. the ratio of rotation period to convective turnover time) of 76 of these stars from stellar structure- and evolution-models for pre-main sequence stars.

Results. The young stars in IC 348 show no correlation between X-ray activity and rotation period. For the Rossby numbers, nearly all IC 348 stars are in the saturated regime of the activity-rotation relation defined by main-sequence stars. Searching for possible super-saturation effects, we find a marginal (but statistically in-significant) trend that the stars with the smallest Rossby numbers have slightly lower X-ray activity levels. There are no significant differences in the X-ray activity level for stars of different spectral types and no relation between spectral type and Rossby number is seen. In addition, for stars belonging to different IR-classes, no significant differences are present for the X-ray activity level as well as for their Rossby numbers. We compare the dispersion in the fractional X-ray luminosities of the stars in the saturated rotation regime in IC 348 to that seen in younger and older stellar populations. The scatter seen in the $\approx 3$ Myr old IC $348\left[\sigma\left(\log \left(L_{\mathrm{X}} / L_{\mathrm{bol}}\right)\right)=0.43\right]$ is considerably smaller than for the $\approx 1$ Myr old Orion nebula cluster $\left[\sigma\left(\log \left(L_{\mathrm{X}} / L_{\mathrm{bol}}\right)\right)=0.63\right]$, but, at the same time, considerably larger than the dispersion seen in the $\approx 30$ Myr old cluster NGC 2547 $\left[\sigma\left(\log \left(L_{\mathrm{X}} / L_{\mathrm{bol}}\right)\right)=0.24\right]$ and in main-sequence stars.

Conclusions. The results of our X-ray analysis of IC 348 show that neither the rotation rates nor the presence/absence of circumstellar disks are of fundamental importance for determining the level of X-ray activity in TTS. Our results suggest that the scatter in the X-ray activity levels for the rapidly rotating members of young clusters decreases with the age of the stellar population. We interpret this as a signature of the changing interior structure of pre-main sequence stars and the consequent changes in the dynamo mechanisms that are responsible for the magnetic field generation.
\end{abstract}

Key words. circumstellar matter - stars: pre-main sequence - stars: activity - stars: magnetic field

\section{Introduction}

Young stellar objects (YSOs) in all evolutionary stages, from class I protostars, to T Tauri stars (TTS) to zero-age mainsequence stars, have highly $\left(\sim 10^{3}-10^{4}\right.$ times $)$ elevated levels of $\mathrm{X}$-ray activity (for reviews of the X-ray properties of YSOs and stellar coronal astronomy in general, see Feigelson \& Montmerle 1999; Favata \& Micela 2003). Despite many years of research, the physical origin of this X-ray activity remains poorly understood. Although there is strong evidence that in most TTS the $\mathrm{X}$-ray emission originates from magnetically confined coronal plasma (e.g., Preibisch et al. 2005), it is unclear what kind of dynamo processes create the required magnetic fields.

For main-sequence stars, the level of the magnetic activity (and thus the strength of the X-ray emission) is mainly determined by their rotation rate. Observations have revealed a clear rotation-activity relation of the form $L_{\mathrm{X}} / L_{\mathrm{bol}} \propto P_{\mathrm{rot}}^{-2.6}$ (e.g., Pallavicini et al. 1981; Pizzolato et al. 2003), which is in good agreement with the expectations of solar-like $\alpha-\Omega$ dynamo models (e.g., Maggio et al. 1987; Ossendrijver 2003). The solar dynamo is thought to be anchored in the "tachocline", a thin zone between the inner radiative core and the outer convection zone. However, the connection between the observed surface magnetic activity and the properties of the dynamo generating the magnetic flux is not yet fully understood (e.g., Iş1k et al. 2011).
For main-sequence stars, the increase in magnetic activity towards shorter rotation periods stops for periods shorter than $\sim 2-$ 3 days, where the activity saturates around $\log \left(L_{\mathrm{X}} / L_{\mathrm{bol}}\right) \approx-3$. The physical reasons for this saturation effect are poorly understood (see, e.g., Jardine \& Unruh 1999). For extremely rapidly rotating stars, the activity level seems to decrease slightly with increasing rotation rate (Prosser et al. 1996; Randich 2000; James et al. 2000; Jeffries et al. 2011), a phenomenon that is denoted as "super-saturation" and also not well-understood.

Studies of stellar populations with different ages show that that there is a continuous evolution from the very high $\mathrm{X}$-ray activity levels in the youngest stages to the much lower activity seen in older (more than a few hundred Myr old) stars (e.g., Güdel et al. 1997; Preibisch \& Feigelson 2005; Telleschi et al. 2005). This evolution can be explained by the temporal decrease in the stellar angular momentum (Bouvier et al. 1997; Herbst et al. 2007). Furthermore, the presence of strong magnetic fields on the surface of T Tauri stars has been clearly established (e.g., Johns-Krull 2007). These pieces of evidence suggest that the $\mathrm{X}$-ray activity of YSOs originates in dynamo processes similar to those present in our Sun.

However, the relation between rotation and X-ray activity in TTS remained unclear until recently, since in most studies of star forming regions the number of X-ray detected TTS with known rotation periods was too small to draw sound conclusions. 
A few years ago, the Chandra Orion Ultradeep Project (COUP), a ten-day long observation of the Orion nebula cluster (ONC) with Chandra/ACIS (for details of the observation and data analysis, see Getman et al. 2005) and the XMM-Newton Extended Survey of the Taurus Molecular Cloud (XEST, see Güdel et al. 2007) provided very sensitive X-ray data sets for large samples of TTS. The COUP and the XEST data have both shown that the TTS in Orion and Taurus do not follow the relation between rotation period and X-ray luminosity for mainsequence stars (Preibisch et al. 2005; Briggs et al. 2007). In addition the TTS spin up during the first $\sim 10-30$ Myr (Herbst \& Mundt 2005) does not lead to an increase in the X-ray luminosity. This places doubt on the solar-like dynamo activity scenario for TTS. Another argument against solar-like dynamos in young TTS comes from theoretical considerations: at ages of $\leq 2 \mathrm{Myr}$, most TTS are expected to be fully convective, and thus should not possess a tachocline. The conventional $\alpha-\Omega$ dynamo cannot work in such a situation. Several alternative dynamo concepts have been developed for fully convective stars (e.g., Giampapa et al. 1996; Küker \& Rüdiger 1999; Dobler et al. 2006; Chabrier \& Küker 2006; Browning \& Basri 2007; Vögler \& Schüssler 2007; Pietarila Graham et al. 2010). Although the reliability of these theoretical models is not entirely clear, there is good evidence for the simultaneous presence of an $\alpha-\Omega$ dynamo and some kind of a small-scale turbulent dynamo in the convection zone of our Sun (e.g., Durney et al. 1993; Trujillo Bueno et al. 2004).

Direct observations of the relation between rotation and magnetic activity for stellar samples spanning a wide range of ages can provide fundamental constraints on the theoretical models. Numerous large datasets are available for samples of main-sequence stars as well as for young stellar clusters with ages as young as 30 Myr (e.g., Prosser et al. 1996; Stauffer et al. 1997a,b; Randich 2000; Jeffries et al. 2011). For younger ages, however, there is still a clear lack of reasonably large stellar samples for which good activity and rotation data are available. The data obtained in the COUP and XEST observations do both indicate that there are very young stellar populations of only $\lesssim 1 \mathrm{Myr}$ old (see, e.g., Weights et al. 2009; Dib et al. 2010; Luhman et al. 2010). Since the stellar rotation, the magnetic activity levels, and other basic stellar parameters evolve strongly in the age range between 1 Myr and $30 \mathrm{Myr}$, a sample of stars with an age of a few Myr can provide valuable information.

With an age of $\approx 3$ Myr (Luhman et al. 2003; Mayne et al. 2007), the young cluster IC 348 is very well-suited in this respect. This age is particularly interesting because it corresponds to the point in time when the structure of solar-mass stars changes from a fully convective interior to a radiative core plus convective envelope structure, and this should affect the dynamo processes that are the ultimate source of the magnetic activity.

\section{X-ray and rotation data for IC 348}

IC 348 is the nearest $(\approx 310$ pc, Herbig 1998$)$ rich and compact very young stellar cluster (Herbst 2008). In a large number of observational studies, more than 300 individual cluster members have been identified and accurately characterized using optical and infrared spectroscopy and photometry (Herbig 1954, 1998; Lada \& Lada 1995; Luhman et al. 1998; Muench et al. 2003; Preibisch et al. 2003; Luhman et al. 1998; Luhman 1999; Luhman et al. 2003, 2005. For nearly all of these stars, basic parameters such as luminosity, effective temperature, mass, and age are known. The mean age of the cluster members is

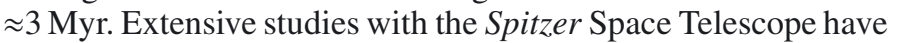

provided comprehensive information about the frequency and nature of the circumstellar disk population in IC 348 (Lada et al. 2006; Muench et al. 2007; Currie \& Kenyon 2009).

\subsection{Chandra X-ray observations}

We targeted IC 348 during four different observations with the Imaging Array of the Chandra Advanced CCD Imaging Spectrometer (ACIS-I). The first observation was obtained in September 2000 (ObsID 606, 52.9 ks exposure time, PI: Th. Preibisch), and the results were presented in Preibisch \& Zinnecker (2001) and Preibisch \& Zinnecker (2002). Three additional observations were performed in March 2008 (ObsID 8584, 50.1 ks exposure time, PI: N. Calvet, ObsID 8933, 40.1 ks exposure time, PI: S. Wolk, and ObsID 8944, 38.6 ks exposure time, PI: S. Wolk). While ObsID 606 was centered at the optical cluster center, ObsID 8584 is shifted by $5.6^{\prime}$ to the south-west, and ObsIDs 8944 and 8944 are shifted 13.1' to the south-west. The total area covered by the combined Chandra data set is about 555 square-arcmin and includes most known cluster members.

For the study presented in this paper, we merged and analyzed all four Chandra data sets to determine the X-ray luminosities of the TTS in IC 348. The source detection in the merged image was performed in a standard way by using the wavdetect algorithm in CIAO to locate X-ray sources in our merged image. The resulting preliminary source list was extended by adding additional possible sources identified by visual inspection. This yielded a catalog of 392 possible X-ray sources. To clean this catalog of spurious sources and determine the properties of the $\mathrm{X}$-ray sources, we performed a detailed analysis of each individual candidate source with the ACIS Extract software package $^{1}$ (Broos et al. 2010), following the procedures described in Getman et al. (2005), Townsley et al. (2003), and Broos et al. (2007). The final catalog contains 290 X-ray sources.

Intrinsic, i.e. extinction-corrected, X-ray luminosities for the sources were determined using the XPHOT software ${ }^{2}$, developed by Getman et al. (2010), assuming a distance of $310 \mathrm{pc}$. For sources with fewer than four net counts (for which XPHOT does not work), an estimate of the X-ray luminosity was derived from the FLUX2 values computed by ACIS Extract and the median energy of the detected photons. For those cluster members that were not detected as X-ray sources in the Chandra data, we determined upper limits to their X-ray luminosities in the following way. First, extraction regions were defined as the $90 \%$ contours of the local PSF and then the number of counts in the target aperture and an estimate of the local background was determined with ACIS Extract. Upper limits to the number of source counts were then calculated with the Bayesian method to determine confidence intervals involving Poisson-distributed data described in Kraft et al. (1991), using a confidence level of 0.9. To obtain upper limits to the X-ray luminosity, these source count upper limits were devided by the local exposure time and multiplied with the mean conversion factor between the count rate and the X-ray luminosity derived for the X-ray detected TTS from our IC 348 sample.

A general description of the resulting X-ray properties and an investigation of relations to the basic stellar parameters is given in Stelzer et al. (2012).

\footnotetext{
1 http://www . astro.psu.edu/xray/docs/TARA/ ae_users_guide.html

2 http://WwW.astro.psu.edu/users/gkosta/XPHOT/
} 


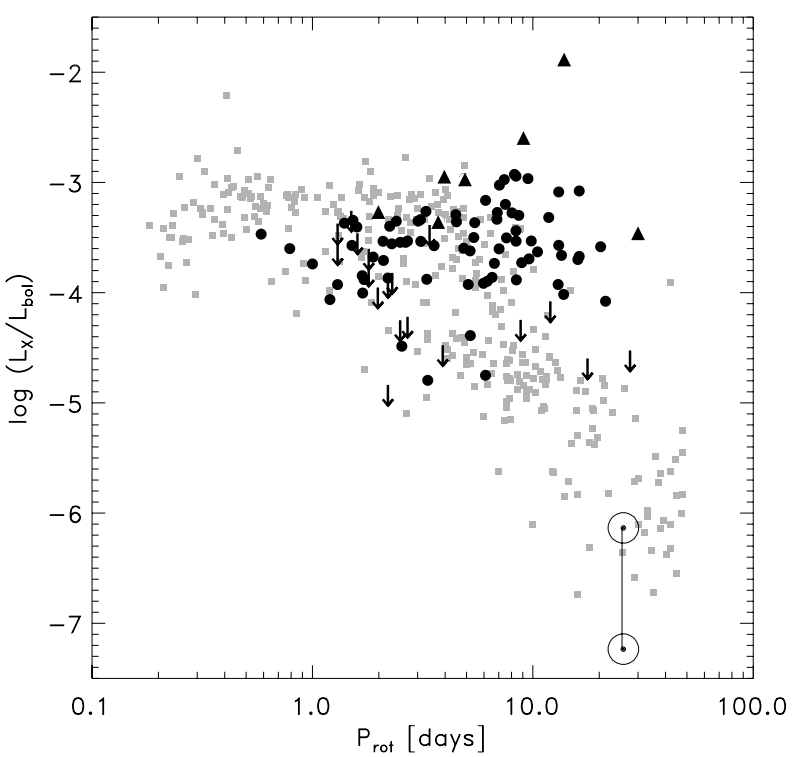

Fig. 1. Fractional X-ray luminosity versus rotation period. This plot compares the IC348 TTS (solid black dots) to MS stars from Pizzolato et al. (2003) and Messina et al. (2003) (gray boxes) and the Sun. IC 348 sources with strong flares during the Chandra observation are marked by triangles.

\subsection{Rotation periods of IC 348 stars from the literature}

Numerous determinations of rotation periods have been performed in the past few years for stars in IC 348 starting with Herbst et al. (2000). From the studies of Cohen et al. (2004), Littlefair et al. (2005), Kızıloğlu et al. (2005), Cieza \& Baliber (2006), and Nordhagen et al. (2006), we collected a catalog with rotational periods for 206 stars in IC 348. For most stars that are listed in more than one of these studies, the rotation periods agreed to within typically a few percent. We used the mean value of the listed rotation periods in these cases. In a few cases of significant discrepancies, we calculated the mean after dropping the outlying value.

\subsection{Construction of the rotation-activity catalog}

The starting point for the construction of the rotation-activity catalog was the membership list for IC 348 from Luhman et al. (2003), which contains stellar parameters such as bolometric luminosity, spectral type, and effective temperature for 288 members. This sample is thought to be complete for $A_{\mathrm{V}} \leq 4 \mathrm{mag}$ and masses of $M \geq 0.03 M_{\odot}$. For each star with known rotation period, we searched for an associated X-ray source within $\approx 2^{\prime \prime}$ of the catalogued optical position. For the 18 cluster members with known rotation periods that are located in the Chandra area but not detected as X-ray sources, we used the upper limits to the $\mathrm{X}$-ray luminosity as described above whereas stellar parameters were taken from Luhman et al. (2003) and Muench et al. (2007).

Our final rotation-activity catalog consists of 82 stars with measured X-ray luminosities and 18 stars with upper limits to the X-ray luminosity. Tables 2 and 3 list the stellar parameters and X-ray luminosities for the 100 stars in our final sample.

\section{X-ray activity and rotation periods}

In Fig. 1, we show the fractional X-ray luminosities $L_{\mathrm{X}} / L_{\mathrm{bol}}$ versus rotation periods for the TTS in IC 348 and compare them to data for main-sequence stars. The periods for the stars in our sample range from 0.585 days to 30 days. It is obvious that the IC 348 stars do not follow the well-established activity-rotation relation defined by the main-sequence stars, i.e. increasing activity for decreasing rotation periods followed by saturation at $L_{\mathrm{X}} / L_{\mathrm{bol}} \sim 10^{-3}$ for periods shorter than about three days. Instead, the IC 348 stars show no relation between $\mathrm{X}$-ray activity and rotation period. To identify stars that displayed large X-ray flares during the Chandra observation, we inspected the X-ray lightcurves. Stars for which the amplitude of the count rate variation in the lightcurve is higher than 10 are marked by triangles in Fig. 1. We note that the two stars with the highest fractional X-ray luminosities $\left(\log \left(L_{\mathrm{X}} / L_{\mathrm{bol}}\right)=-2.6\right.$ for star J $034427.02+320443.6$ and $\log \left(L_{\mathrm{X}} / L_{\mathrm{bol}}\right)=-1.9$ for star J 034359.69+321402.9) showed strong X-ray flares during the Chandra observation; the amplitude of the count rate variation was 29.6 and 36.5, respectively, and the exponential decay time of the flares was $\sim 4-6 \mathrm{ks}$. Therefore, the effect of these short flares on the mean values of X-ray luminosity averaged over the entire duration of the observation is small ${ }^{3}$.

The rotation periods of the X-ray undetected stars are rather uniformly distributed across the full range of periods for the $\mathrm{X}$-ray detected stars. This implies that the X-ray detection limit does not produce a systematic bias against either very rapid or slow rotators.

The data show that the X-ray activity level for the TTS in IC 348 seems to be independent of the rotation period. For the rapid rotators (periods $\leq 5$ days), the absence of an activityrotation correlation may be an effect of the saturation seen in main-sequence stars. However, there is a considerable number of rather slowly rotating IC 348 stars (periods $\gtrsim 10$ days) that have much higher X-ray activity levels than similarly slowly rotating main-sequence stars. We can thus confidently conclude that the IC 348 TTS do not follow the same relation between rotation period and fractional $\mathrm{X}$-ray luminosity as seen for main-sequence stars.

\section{X-ray activity and Rossby numbers}

Although the efficiency of the magnetic field generation in the $\alpha-\Omega$ dynamo model increases with the rotation rate, there is no direct causal relationship. The dynamo number depends instead on the radial gradient of the angular velocity and the characteristic scale length of convection at the base of the convection zone (see Ossendrijver 2003). A detailed theoretical analysis shows that the dynamo number scales as the inverse square of the Rossby number Ro (e.g., Maggio et al. 1987), which is defined as the ratio of the rotation period to the convective turnover time $\tau_{\mathrm{c}}$, i.e. $R o:=P_{\text {rot }} / \tau_{\mathrm{c}}$. This theoretical prediction is well-confirmed by data for main-sequence stars, which follow a considerably tighter relationship between magnetic activity and Rossby number than magnetic activity and rotation period (e.g., Montesinos et al. 2001; Pizzolato et al. 2003).

\footnotetext{
3 A detailed analysis of the effects of flares on the mean X-ray luminosity of TTS was performed in the context of the Chandra Orion Ultradeep Projects (Getman et al. 2005). An analysis of the X-ray lightcurves was used to derive the "characteristic" X-ray luminosities by removing the effect of strong flares. It was found that the difference between these "characteristic" X-ray luminosities and the average $\mathrm{X}$-ray luminosities (i.e. the mean values determined without removing flares) is very small and does not significantly affect the relations between X-ray and basic stellar properties (Preibisch et al. 2005). Our IC 348 Chandra observations have long enough exposure times (compared to the typical short duration of a flare) for individual flares to have only very minor effects on the average X-ray luminosities.
} 


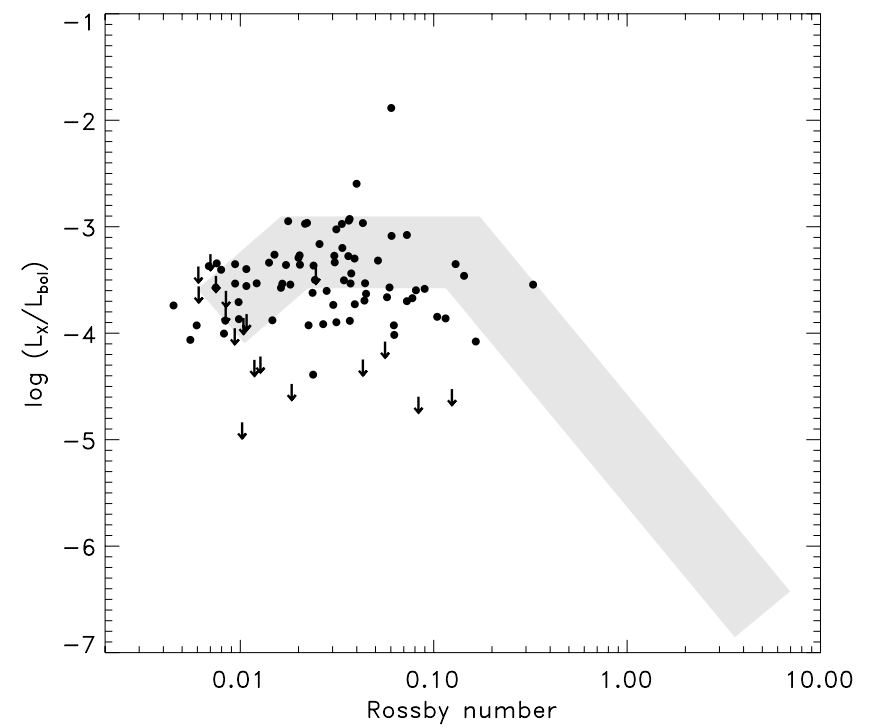

Fig. 2. Fractional X-ray luminosity versus Rossby number for the IC 348 stars. Black arrows indicate upper limits on the X-ray activity. The gray shaded area shows the relation and the width of its typical scatter found for MS stars (from Pizzolato et al. 2003).

Numerous studies have confirmed that for slowly rotating stars, activity rises as $L_{\mathrm{X}} / L_{\mathrm{bol}} \propto R o^{-2}$. Saturation at the $L_{\mathrm{X}} / L_{\mathrm{bol}} \approx$ $10^{-3}$ level is reached around $R o \sim 0.1$, which is followed by a regime of "supersaturation" for very small Rossby numbers, $R o \lesssim 0.02$ (e.g., Randich 2000; Jeffries et al. 2011).

\subsection{Determination of convective turnover times for the TTS}

The convective turnover timescale $\tau_{\mathrm{c}}$ is a sensitive function of the physical properties in the stellar interior. For main-sequence stars, semi-empirical interpolations of $\tau_{\mathrm{c}}$ values as a function of the color or the stellar mass are available and provide an easy way to estimate the Rossby numbers. For TTS, which have a considerably different and rapidly evolving internal structure, the situation is far more complex because the $\tau_{\mathrm{c}}$ values depend strongly on age. The convective turnover timescales for the young TTS are several times longer than for main-sequence stars of the same mass. Detailed stellar evolution model calculations are required to determine reliable convective turnover times for TTS in a self-consistent way.

Model calculations of this type were performed for the analysis of the activity-rotation relation in the Orion nebula cluster described in Preibisch et al. (2005). First, a large set of stellar models was computed with the Yale Stellar Evolution Code (see Kim \& Demarque 1996) for stellar masses between $0.065 M_{\odot}$ and 4.0 $M_{\odot}$. In the second step, the convective turnover time for each star in the observed sample was determined from the model that reproduced its observed effective temperature and luminosity. This resulted in a database of 562 different $\left(T_{\mathrm{eff}}, L_{\mathrm{bol}}, \tau_{\mathrm{c}}\right)$ values.

We used these results to determine convective turnover times for the TTS in IC 348 by interpolating their stellar parameters $T_{\text {eff }}$ and $L_{\text {bol }}$ against those in the database to determine $\tau_{\mathrm{c}}$. This resulted in Rossby numbers for 76 stars in our sample.

\subsection{The activity-Rossby number relation for the TTS}

Rossby numbers were computed by dividing the rotation periods of the stars by the values for their local convective turnover time derived above. Figure 2 shows the fractional X-ray luminosities of the TTS versus the resulting Rossby numbers. The plot shows

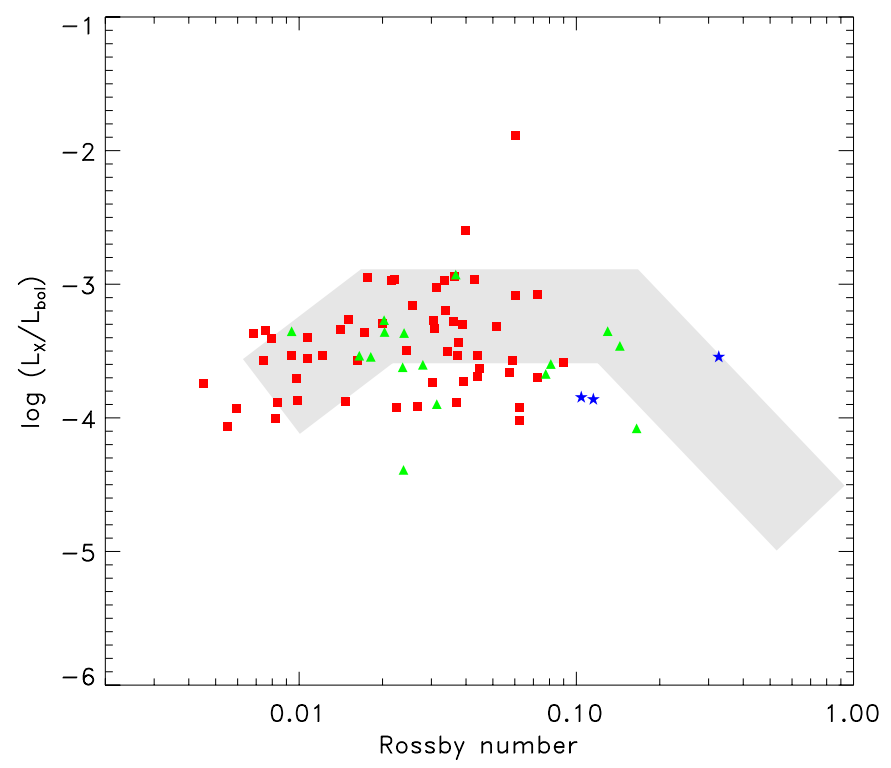

Fig. 3. Comparison of fractional X-ray luminosity versus Rossby number for stars in IC 348 for the different spectral types G (blue asterisks), $\mathrm{K}$ (green triangles), and $\mathrm{M}$ (red squares). The gray shaded area shows the relation and the width of its typical scatter found for MS stars (from Pizzolato et al. 2003).

no strong relation between these two quantities as expected for such low Rossby numbers. Nearly all TTS have Rossby numbers $<0.2$ and are therefore in the saturated or super-saturated regime of the activity-Rossby number relation for main-sequence stars.

To search for indications of super-saturation, we compared the fractional X-ray luminosities of the TTS in the saturated $(0.1>R o>0.02)$ and super-saturated $(R o \leq 0.02)$ regimes. We found nearly equal median values of $\log \left(L_{\mathrm{X}} / L_{\mathrm{bol}}\right)=-3.51$ for the TTS in the saturated regime and -3.56 for those in the super-saturated regime; thus, the stars from IC 348 show no clear evidence of supersaturation. Nevertheless, there may be a hint of supersaturation if we consider only stars with very small Rossby numbers of $R o \leq 0.006$.

All three stars in this range have remarkably low activity levels of between -3.7 and -4.1 . If this is a real effect, the border for supersaturation would be shifted by about a factor of three relative to the border determined by Randich (1998) for mainsequence stars.

A difference between TTS and main-sequence stars manifests itself in the wide dispersion of fractional X-ray luminosities at a given Rossby number among the TTS. The activity levels scatter over more than one order of magnitude, in remarkable contrast to the much smaller scatter found for main-sequence stars in the saturated rotation regime (see Pizzolato et al. 2003). This result suggests that additional factors, other than the rotation period, are important for determining the level of X-ray activity in TTS.

\subsection{The activity-Rossby number relation for stars of different spectral types and infrared-classes}

A comparison of $L_{X} / L_{\text {bol }}$ versus Rossby numbers for different spectral classes is shown in Fig. 3.

We found no statistically significant difference between the median values of the fractional luminosity for the different spectral types. In addition, the ranges of Rossby numbers for the different spectral types seem to be quite similar. The three G-type 


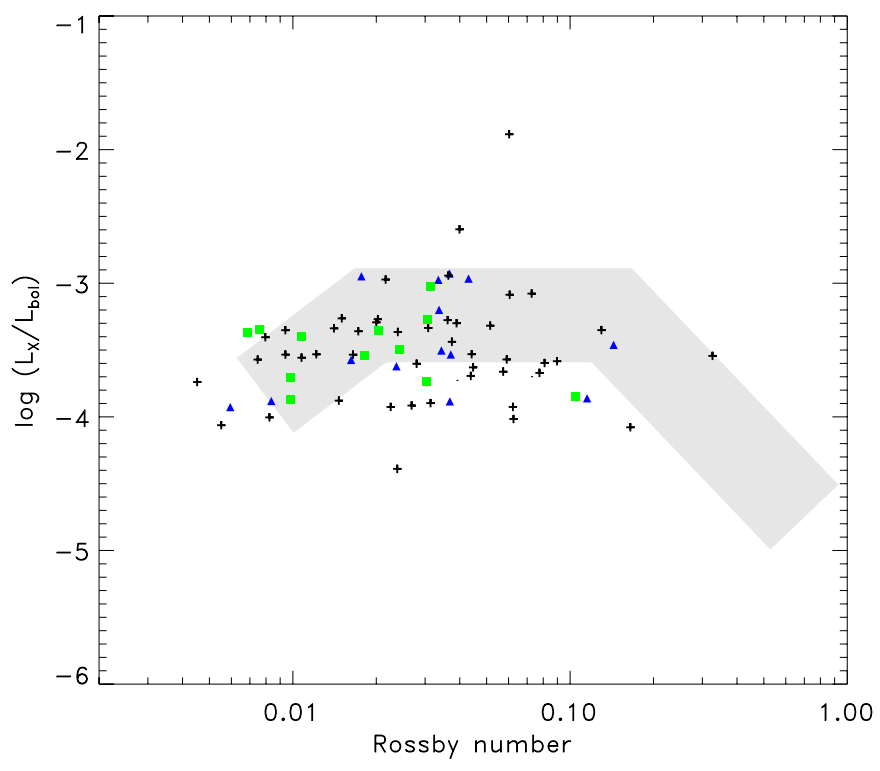

Fig. 4. Comparison of fractional X-ray luminosity versus Rossby number for stars in IC 348 for the different IR-classes II (blue triangles), II/III (green squares), and III (black crosses). The gray shaded area shows the relation and the width of its typical scatter found for MS stars (from Pizzolato et al. 2003).

stars have larger Rossby numbers than the K- and M-type stars, although this may simply be an effect of small-number statistics.

In Fig. 4, we show X-ray activity versus Rossby number for the different infrared-classes, which trace the circumstellar material around the young stars. We used the infrared-classes derived by Lada et al. (2006) from the observed Spitzer/IRAC spectral energy distributions (SEDs) between $3.6 \mu \mathrm{m}$ and $8 \mu \mathrm{m}$. Objects with an SED slope of $\alpha_{3-8}>-0.5$ are class 1 and are thought to be very young stellar objects surrounded by circumstellar disks and envelopes. Class II objects $\left(-0.5 \geq \alpha_{3-8} \geq\right.$ $-1.8)$ are stars with thick circumstellar disks. Class II/III objects $\left(-1.8 \geq \alpha_{3-8} \geq-2.56\right)$ are thought to be stars surrounded by "anemic" disks, whereas class III objects $\left(\alpha_{3-8}<-2.56\right)$ are disk-less stars. The "anemic" disk objects are probably transition objects that have either optically thin disks, or disks with large inner holes (see Lada et al. 2006).

The plot shows no obvious differences in either the X-ray activity levels or the Rossby numbers of the different infrared classes. This suggests that the presence or absence and the properties of the circumstellar material are independent of the level of $\mathrm{X}$-ray emission. This is consistent with the results derived from Chandra observations of the Orion nebula cluster (Preibisch et al. 2005).

Investigating the plot of rotation periods versus fractional luminosities for the different infrared classes, we found no significant relation. We also found no indications of a relation between the Rossby number and the infrared class.

\section{Comparison of IC 348 to young clusters of different age}

During the pre-main sequence lifetime of young stars, several important changes occur with respect to the level and origin of the magnetic activity. X-ray studies of young clusters with different age have shown that the level of X-ray activity is approximately constant during the initial $\sim 10 \mathrm{Myr}$, and starts to decline significantly at ages above $\sim 10-30 \mathrm{Myr}$
Table 1. Statistical values (mean, standard deviation, median, and median absolute deviation) for the activity level $\log \left(L_{\mathrm{X}} / L_{\mathrm{bol}}\right)$ of young stars in the Orion nebula cluster, IC 348, and NGC 2547.

\begin{tabular}{lrcc}
\hline \hline Stat. value & Orion & IC 348 & NGC 2547 \\
\hline Mean & -3.39 & -3.53 & -3.20 \\
Std. Dev. & 0.63 & 0.43 & 0.24 \\
Median & -3.31 & -3.53 & -3.23 \\
MAD & 0.39 & 0.21 & 0.18 \\
\hline
\end{tabular}

(Preibisch \& Feigelson 2005). Another important aspect is that the internal structure of the young stars changes from a fully convective structure to a radiative core plus convective envelope structure. The timescale for these transitions depends on the stellar mass; for stars with masses of around one solar mass, this change occurs at ages around 2-4 Myr. It is therefore interesting to compare the rotation-activity relation for IC 348 to that seen in other young clusters of different age. While there is certainly no lack of X-ray data on young stellar clusters, rotation data are often unavailable for a sufficiently large number of cluster members for statistical studies, especially for the particularly interesting age period of $\leq 10$ Myr. Another serious obstacle is often the non-availability of reliable Rossby numbers for young pre-main sequence stars.

Here we consider two other young clusters for which similarly good X-ray and rotation data as well as reliable Rossby numbers are available for a sufficiently large number of stars. The first data set is the sample of TTS in the $\approx 1$ Myr old Orion Nebula Cluster from Preibisch et al. (2005). The second sample is taken from the recent study of the $\approx 30$ Myr old cluster NGC 2547 by Jeffries et al. (2011).

In Fig. 5, we compare the relations between X-ray activity and Rossby numbers for the three studied clusters. The three samples are similar in the sense that no correlation can be seen between X-ray activity and Rossby number. A striking difference is however seen in the scatter in the X-ray activity levels for the stars in the saturated Rossby number regime. Table 1 lists statistical values for the scatter in the activity levels seen in each sample. The scatter is largest in the Orion nebula cluster sample $\left[\sigma\left(\log \left(L_{\mathrm{X}} / L_{\mathrm{bol}}\right)\right)=0.63\right]$, but considerably smaller in IC $348\left[\sigma\left(\log \left(L_{\mathrm{X}} / L_{\mathrm{bol}}\right)\right)=0.43\right]$, and yet smaller in the case of NGC $2547\left[\sigma\left(\log \left(L_{\mathrm{X}} / L_{\text {bol }}\right)\right)=0.24\right]$. We note that the scatter seen in NGC 2547 is representative of the scatter seen in mainsequence stars (see Pizzolato et al. 2003). As the age of the Orion nebula cluster sample is $\approx 1 \mathrm{Myr}$, whereas IC 348 is $\approx 3$ Myr old, and NGC 2547 is $\approx 30$ Myr old, we find a decrease in the scatter in the X-ray activity levels for stars in the saturated Rossby number regime with the age of the stellar population.

\section{Conclusions}

Our analysis of the relation between X-ray activity (as traced by deep Chandra observations) and the rotation properties of the TTS in the young cluster IC 348 has yielded the following results. First, we have shown that there is no correlation between the fractional X-ray luminosity and the rotation periods of the TTS; even the rather slowly rotating stars (periods $\gtrsim 10$ days) display very strong X-ray activity. Second, according to the Rossby numbers (that we have determined based on detailed stellar models for pre-main sequence stars), all TTS are in the saturated regime of the rotation-activity relation defined by main-sequence stars. Third, we have found no significant evidence of super-saturation among the most rapid rotators, 
Table 2. Stellar parameters for the sample of stars in IC 348.

\begin{tabular}{|c|c|c|c|c|c|c|}
\hline$\overline{\mathrm{RA}, \mathrm{Dec}}$ & $\begin{array}{c}L_{\text {bol }} \\
L_{\odot} \\
\end{array}$ & Spectral type & $\begin{array}{c}\text { Turnover time } \\
\text { days }\end{array}$ & $\begin{array}{c}\begin{array}{c}\text { Period } \\
\text { days }\end{array} \\
\end{array}$ & Class & $\begin{array}{c}\text { X-ray luminosity } \\
\text { erg/s }\end{array}$ \\
\hline $034424.57+320357.1$ & 0.44 & M1 & 227.7 & 4.92 & III & $1.80 \times 10^{30}$ \\
\hline $034422.57+320153.7$ & 0.51 & M2.5 & 221.4 & 1.00 & III & $3.57 \times 10^{29}$ \\
\hline $034421.26+320502.4$ & 0.32 & M2.5 & 223.2 & 6.87 & III & $5.70 \times 10^{29}$ \\
\hline $034422.29+320542.7$ & 0.54 & K8 & 209.0 & 30.00 & II & $7.18 \times 10^{29}$ \\
\hline $034421.66+320624.8$ & 0.29 & M2.75 & 223.7 & 8.38 & III & $4.06 \times 10^{29}$ \\
\hline $034411.26+320612.1$ & 0.39 & M0 & 234.9 & 10.50 & III & $3.52 \times 10^{29}$ \\
\hline $034420.02+320645.5$ & 0.12 & M3.5 & 221.6 & 8.63 & III & $2.32 \times 10^{29}$ \\
\hline $034406.79+320754.1$ & 0.17 & M4.25 & 218.6 & 1.30 & II & $7.74 \times 10^{28}$ \\
\hline $034411.22+320816.3$ & 0.14 & M5.25 & 209.3 & 13.02 & III & $6.38 \times 10^{28}$ \\
\hline $034423.67+320646.5$ & 0.39 & M2.5 & 222.7 & 9.83 & III & $4.42 \times 10^{29}$ \\
\hline $034405.00+320953.8$ & 0.93 & $\mathrm{~K} 3.5$ & 129.5 & 21.37 & III & $2.99 \times 10^{29}$ \\
\hline $034419.24+320734.7$ & 0.26 & M3.75 & 221.0 & 7.60 & II & $3.14 \times 10^{29}$ \\
\hline $034416.43+320955.2$ & 1.4 & K0 & 23.2 & 3.01 & III & $2.40 \times 10^{30}$ \\
\hline $034421.56+321017.4$ & 0.29 & M1.5 & 224.8 & 7.05 & II/III & $1.05 \times 10^{30}$ \\
\hline $034421.91+321211.6$ & 0.29 & M4 & 220.6 & 13.79 & III & $1.07 \times 10^{29}$ \\
\hline $034422.32+321200.8$ & 0.33 & M1 & 228.4 & 8.42 & II & $1.66 \times 10^{29}$ \\
\hline $034422.98+321157.3$ & 0.22 & M2.25 & 225.9 & 5.09 & III & $1.00 \times 10^{29}$ \\
\hline $034501.52+321051.5$ & 0.94 & K0 & - & 1.89 & III & $7.60 \times 10^{29}$ \\
\hline $034425.58+321130.5$ & 0.34 & M0 & 237.6 & 6.1 & - & $8.99 \times 10^{29}$ \\
\hline $034426.69+320820.3$ & 0.53 & M0.5 & 227.7 & 8.90 & - & $3.81 \times 10^{29}$ \\
\hline $034433.31+320939.6$ & 0.41 & M2 & 223.8 & 2.20 & II/III & $2.14 \times 10^{29}$ \\
\hline $034427.88+320731.6$ & 0.46 & M2 & 222.5 & 5.97 & III & $2.15 \times 10^{29}$ \\
\hline $034432.77+320915.8$ & 0.36 & M3.25 & 221.7 & 5.39 & II/III & $4.38 \times 10^{29}$ \\
\hline $034432.58+320855.8$ & 0.47 & M3 & 221.2 & 6.69 & II/III & $3.33 \times 10^{29}$ \\
\hline $034432.74+320837.5$ & 4.4 & G6 & 8.1 & 2.64 & III & $4.84 \times 10^{30}$ \\
\hline $034433.98+320854.1$ & 0.56 & M0 & 223.3 & 16.23 & III & $1.80 \times 10^{30}$ \\
\hline $034428.47+320722.4$ & 0.71 & K6.5 & 251.3 & 7.02 & III & $6.82 \times 10^{29}$ \\
\hline $034441.31+321025.3$ & 0.17 & M4.75 & 216.0 & 3.72 & III & $2.86 \times 10^{29}$ \\
\hline $034439.21+320944.7$ & 0.39 & M2 & 224.4 & 3.97 & II & $1.69 \times 10^{30}$ \\
\hline $034437.41+320900.9$ & 0.51 & M1 & 225.8 & 8.39 & II & $5.75 \times 10^{29}$ \\
\hline $034442.58+321002.5$ & 0.24 & M4.25 & 219.7 & 3.56 & II & $2.46 \times 10^{29}$ \\
\hline $034438.70+320856.7$ & 0.24 & M3.25 & 222.3 & 2.09 & III & $2.70 \times 10^{29}$ \\
\hline $034438.72+320842.0$ & 4.1 & $\mathrm{~K} 3$ & 255.5 & 2.40 & III & $7.02 \times 10^{30}$ \\
\hline $034435.52+320804.5$ & 0.09 & M5.25 & 205.3 & 1.69 & III & $3.43 \times 10^{28}$ \\
\hline $034442.02+320900.1$ & 0.37 & M4.25 & 220.2 & 16.00 & 一 & $2.84 \times 10^{29}$ \\
\hline $034437.89+320804.2$ & 1.4 & K7 & 224.5 & 8.25 & II & $6.36 \times 10^{30}$ \\
\hline $034438.55+320800.7$ & 0.72 & M1.25 & 222.8 & 7.50 & II & $1.75 \times 10^{30}$ \\
\hline $034435.04+320736.9$ & 1.5 & K6.5 & 221.3 & 4.50 & $\mathrm{II} / \mathrm{III}$ & $2.53 \times 10^{30}$ \\
\hline $034438.47+320735.7$ & 1.5 & K6 & 219.6 & 5.19 & II & $1.38 \times 10^{30}$ \\
\hline $034439.25+320735.5$ & 3.6 & K3 & 218.6 & 5.20 & III & $5.65 \times 10^{29}$ \\
\hline $034443.53+320743.0$ & 0.92 & M1 & 222.3 & 8.04 & III & $1.87 \times 10^{30}$ \\
\hline $034436.94+320645.4$ & 17 & G3 & 16.1 & 1.68 & $\mathrm{II} / \mathrm{III}$ & $9.32 \times 10^{30}$ \\
\hline $034434.88+320633.6$ & 0.99 & K5.5 & 227.7 & 5.45 & III & $1.64 \times 10^{30}$ \\
\hline $034442.63+320619.5$ & 0.19 & M1 & 229.2 & 11.80 & III & $3.51 \times 10^{29}$ \\
\hline $034437.41+320611.7$ & 0.51 & K7 & 197.9 & 6.21 & III & $2.49 \times 10^{29}$ \\
\hline $034441.32+320453.5$ & 0.046 & M5 & 199.8 & 1.59 & III & $6.98 \times 10^{28}$ \\
\hline $034425.56+320617.0$ & 0.54 & M2.25 & 221.4 & 7.42 & II & $2.20 \times 10^{30}$ \\
\hline $034427.02+320443.6$ & 0.46 & M1 & 227.0 & 9.06 & III & $4.48 \times 10^{30}$ \\
\hline $034426.63+320358.3$ & 0.39 & M4.75 & 219.4 & 3.09 & III & $6.89 \times 10^{29}$ \\
\hline $034426.03+320430.4$ & 9.9 & G8 & 56.7 & 6.54 & II & $5.24 \times 10^{30}$ \\
\hline $034348.76+320733.4$ & 0.34 & M1.5 & 226.2 & 20.30 & III & $3.41 \times 10^{29}$ \\
\hline $034355.51+320932.5$ & 1.9 & K0 & 60.0 & 4.86 & III & $1.85 \times 10^{30}$ \\
\hline $034349.39+321040.0$ & 0.17 & M3.5 & 216.4 & 13.10 & III & $5.36 \times 10^{29}$ \\
\hline $034423.99+321100.0$ & 3.1 & G0 & - & 2.54 & III & $3.89 \times 10^{29}$ \\
\hline $034359.72+321403.2$ & 0.33 & M0.75 & 229.9 & 13.87 & III & $1.65 \times 10^{31}$ \\
\hline $034404.25+321350.0$ & 0.22 & M4.75 & 217.6 & 1.20 & III & $7.33 \times 10^{28}$ \\
\hline $034417.91+321220.4$ & 0.31 & $\mathrm{M} 2.5$ & 223.5 & 4.47 & III & $6.08 \times 10^{29}$ \\
\hline $034425.57+321230.0$ & 0.45 & M0.5 & 230.3 & 8.38 & III & $1.97 \times 10^{30}$ \\
\hline $034428.12+321600.3$ & 0.3 & M3.25 & 222.2 & 2.70 & III & $3.39 \times 10^{29}$ \\
\hline $034439.81+321804.2$ & 0.39 & M3.75 & 220.8 & 9.50 & II & $1.62 \times 10^{30}$ \\
\hline $034415.58+320921.9$ & 0.018 & M7.5 & - & 0.59 & III & $2.35 \times 10^{28}$ \\
\hline $034438.39+321259.8$ & 0.21 & M0 & 235.2 & 13.49 & III & $1.76 \times 10^{29}$ \\
\hline $034437.79+321218.2$ & 0.19 & M4.5 & 217.9 & 3.27 & III & $3.99 \times 10^{29}$ \\
\hline
\end{tabular}


Table 2. continued.

\begin{tabular}{lcccccc}
\hline \hline Ra, Dec & $\begin{array}{c}L_{\text {bol }} \\
L_{\odot}\end{array}$ & Spectral type & $\begin{array}{c}\text { Turnover time } \\
\text { days }\end{array}$ & $\begin{array}{c}\text { Period } \\
\text { days }\end{array}$ & $\begin{array}{c}\text { Class } \\
\text { X-ray luminosity } \\
\text { erg/s }\end{array}$ \\
\hline $034450.97+321609.6$ & 0.35 & M3.25 & 221.7 & 13.10 & III & $3.62 \times 10^{29}$ \\
$034440.13+321134.3$ & 1.4 & K2 & 98.3 & 1.99 & III & $2.90 \times 10^{30}$ \\
$034441.74+321202.4$ & 0.17 & M5 & 214.4 & 2.10 & II/III & $1.28 \times 10^{29}$ \\
$034448.83+321322.1$ & 0.13 & M2.75 & 225.2 & 6.90 & II/III & $2.67 \times 10^{29}$ \\
$034444.85+321105.8$ & 0.17 & M2.75 & 213.0 & 2.29 & III & $1.81 \times 10^{29}$ \\
$034501.74+321427.9$ & 1.9 & K4 & 209.2 & 16.23 & III & $1.56 \times 10^{30}$ \\
$034450.65+321906.8$ & 135 & A0 & - & 6.10 & III & $9.23 \times 10^{30}$ \\
$034507.61+321028.1$ & 4.9 & G1 & - & 3.34 & III & $3.02 \times 10^{29}$ \\
$034455.63+320920.2$ & 1.4 & K4 & 188.0 & 3.10 & III & $1.57 \times 10^{30}$ \\
$034456.15+320915.5$ & 3.9 & K0 & 137.9 & 2.50 & II/III & $4.29 \times 10^{30}$ \\
$034453.76+320652.2$ & 0.18 & M4 & 219.0 & 9.60 & III & $1.40 \times 10^{29}$ \\
$034456.12+320556.7$ & 0.13 & M2.75 & 225.2 & 3.30 & III & $6.61 \times 10^{28}$ \\
$034505.77+320308.2$ & 0.54 & M0 & 221.4 & 4.9 & - & $2.25 \times 10^{30}$ \\
$034418.26+320732.5$ & 0.078 & M4.75 & 208.5 & 2.24 & II/III & $1.20 \times 10^{29}$ \\
$034423.57+320934.0$ & 0.074 & M5 & 205.1 & 1.72 & II & $3.74 \times 10^{28}$ \\
$034427.28+320717.6$ & 0.038 & M4.75 & 202.2 & 1.53 & II/III & $6.62 \times 10^{28}$ \\
$034431.42+321129.4$ & 0.078 & M5.25 & 203.0 & 1.40 & II/III & $1.28 \times 10^{29}$ \\
$034439.44+321008.2$ & 0.057 & M5 & 202.3 & 1.51 & III & $5.88 \times 10^{28}$ \\
$034351.24+321309.4$ & 4.3 & G5 & - & 0.79 & III & $4.15 \times 10^{30}$ \\
\hline
\end{tabular}

Table 3. Stellar parameters for IC 348 members with upper limits.

\begin{tabular}{lccccc}
\hline \hline RA, Dec & $\begin{array}{c}L_{\text {bol }} \\
L_{\odot}\end{array}$ & Spectral type & $\begin{array}{c}\text { Turnover time } \\
\text { days }\end{array}$ & $\begin{array}{c}\text { Period } \\
\text { days }\end{array}$ & $\begin{array}{c}\text { X-ray luminosity } \\
\text { erg/s }\end{array}$ \\
\hline $034328.22+320159.1$ & 0.768 & M1.75 & 204.5 & 8.8 & $<1.67 \times 10^{29}$ \\
$034345.16+320358.6$ & 0.731 & M0 & 222.2 & 27.6 & $<8.39 \times 10^{28}$ \\
$034359.08+321421.4$ & 0.440 & M3.5 & 212.3 & 17.7 & $<4.28 \times 10^{28}$ \\
$034410.12+320404.4$ & 0.12 & M5.75 & 212.0 & 2.2 & $<6.41 \times 10^{28}$ \\
$034418.19+320959.3$ & 0.15 & M4.25 & 212.5 & 2.7 & $<3.48 \times 10^{28}$ \\
$034420.18+320856.7$ & 0.32 & M2 & 215.4 & 2.2 & $<1.79 \times 10^{28}$ \\
$034421.26+321237.4$ & 0.073 & M4.75 & 213.4 & 2.3 & $<4.24 \times 10^{28}$ \\
$034432.35+320327.3$ & 0.028 & M5.5 & 213.8 & 1.6 & $<3.72 \times 10^{28}$ \\
$034432.80+320413.4$ & 0.065 & M5 & 213.4 & 1.3 & $<6.87 \times 10^{28}$ \\
$034433.79+315830.3$ & 0.492 & M3.75 & 211.6 & 3.9 & $<6.32 \times 10^{28}$ \\
$034434.05+320656.8$ & 0.032 & M7.25 & 138.3 & 3.4 & $<5.06 \times 10^{28}$ \\
$034435.01+320857.4$ & 0.032 & M4.75 & 214.2 & 1.8 & $<2.16 \times 10^{28}$ \\
$034435.44+320856.4$ & 0.16 & M5.25 & 211.5 & 1.98 & $<6.85 \times 10^{28}$ \\
$034435.68+320303.6$ & 0.13 & M3.25 & 214.3 & 12.0 & $<4.17 \times 10^{28}$ \\
$034436.98+320834.0$ & 0.18 & M4.75 & 211.5 & 2.5 & $<3.89 \times 10^{28}$ \\
$034444.23+320847.4$ & 0.040 & M5.75 & 213.5 & 1.8 & $<3.84 \times 10^{28}$ \\
$034444.27+321036.8$ & 0.017 & M5.25 & 214.1 & 1.5 & $<3.61 \times 10^{28}$ \\
$034445.67+321111.0$ & 0.028 & M4.75 & 214.3 & 1.3 & $<4.56 \times 10^{28}$ \\
\hline
\end{tabular}

although our data suggest that stars with extremely low Rossby numbers $(R o \leq 0.006)$ have slightly lower activity levels. This seems to agree with the results of Jeffries et al. (2011), who claim that in their NGC 2547 sample supersaturation occurs only for Rossby numbers lower than 0.005 . In all three of these aspects, the TTS in IC 348 behave similarly to the TTS in the Orion nebula cluster.

A remarkable difference is however seen in the scatter in the X-ray activity levels for the TTS in the saturated rotation regime. The scatter seen in the $\approx 3$ Myr old IC 348 sample is considerably smaller than that in the $\approx 1$ Myr old Orion nebula cluster sample, but, at the same time, considerably larger than seen in the $\approx 30$ Myr old stars in NGC 2547 as well as for mainsequence stars. This suggests that some process reduces the wide distribution of activity levels seen in the youngest stars towards the much narrower distribution in older pre-main sequence and (young) main sequence stars during the first 30 Myr period, whereas the absolute level of the X-ray activity remains roughly constant during that time.

A possible explanation of this effect may be related to the stellar interior structure and the corresponding dynamo mechanisms that are the basis of the magnetic activity that produces the observed X-ray emission. At an age of $\leq 1 \mathrm{Myr}$ (e.g., in the Orion nebula cluster), almost all low- and intermediate mass stars $\left(M \leq 2 M_{\odot}\right)$ are fully convective. As already mentioned, the solar-like $\alpha-\Omega$ dynamo cannot operate in such a situation and some kind of small-scale convective dynamo must be the source of the magnetic activity. The more or less chaotic nature of such a dynamo may be responsible for the very wide scatter in activity levels seen for stars with similar rotation rates and stellar parameters. According to the pre-main sequence stellar evolution models of Siess et al. (2000), a $1.5 M_{\odot}$ star develops a radiative core at an age of about 1 Myr. As time proceeds, stars with increasingly lower masses develop radiative cores (at $\approx 1.7 \mathrm{Myr}$ 

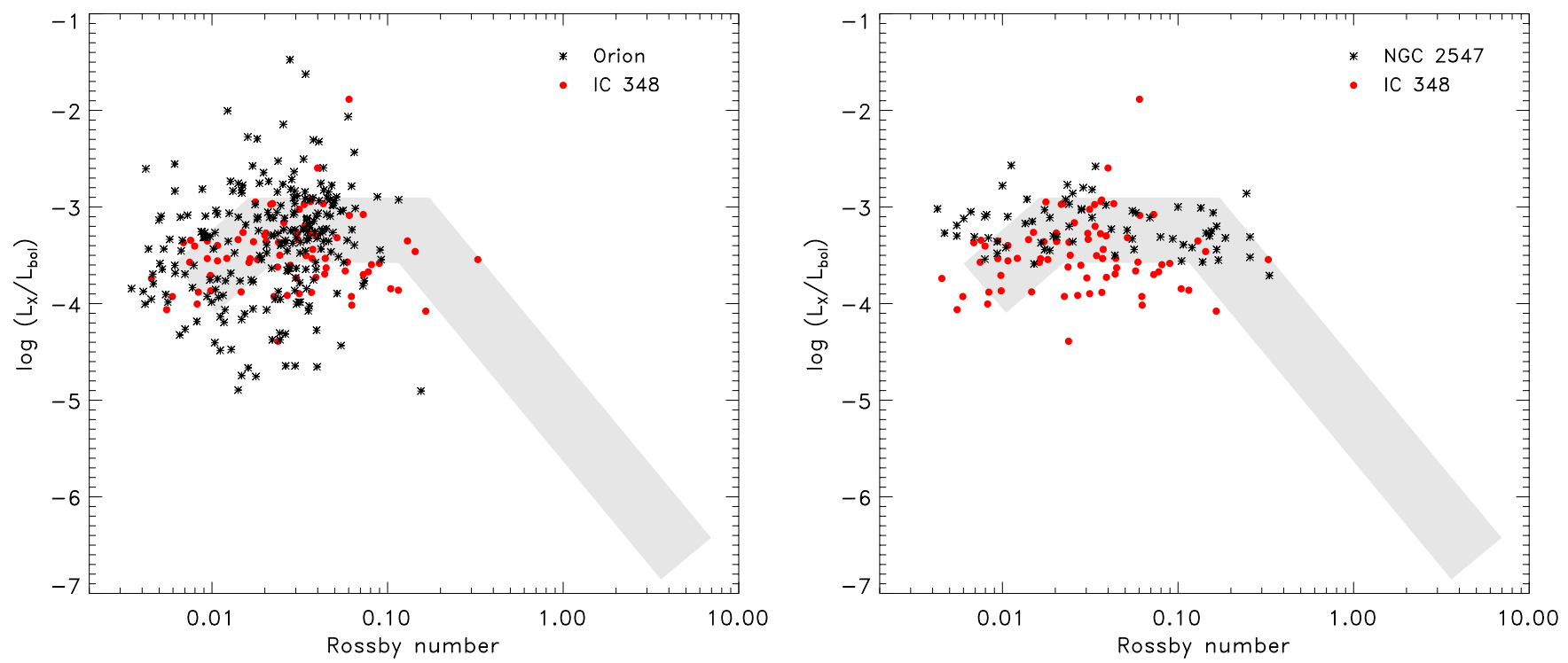

Fig. 5. Fractional X-ray luminosity versus Rossby number for stars in IC 348 (red filled circles) compared to the stars (black asterisks) in the Orion nebula cluster (left) and NGC 2547 (right). The gray shaded area shows the relation and the width of its typical scatter found for MS stars (from Pizzolato et al. 2003).

for $1.2 M_{\odot}$ stars, at $\approx 2.3 \mathrm{Myr}$ for $1.0 M_{\odot}$ stars, and at $\approx 4.2 \mathrm{Myr}$ for $0.8 M_{\odot}$ stars). As soon as a star has a radiative core, the conditions for the operation of a solar-like $\alpha-\Omega$ dynamo are met, which then gradually replaces the convective dynamo. When the total magnetic activity is dominated by the $\alpha-\Omega$ dynamo, stars in the saturated rotation regime have a rather homogeneous level of X-ray activity, i.e. a small scatter in their $L_{\mathrm{X}} / L_{\mathrm{bol}}$ values.

At the 3 Myr age of IC 348 , all stars with $M \geq 0.9 M_{\odot}$ (corresponding to a spectral type of $\mathrm{K} 6$ ) should have a radiative core; this concerns about $25 \%$ of our stellar sample in IC 348 and can explain why the dispersion in the X-ray activity levels in IC 348 is smaller than in the Orion nebula cluster. As time proceeds, an increasing fraction of the lower-mass stars make the transition from a fully convective to a core/envelope structure, where the operation of an $\alpha-\Omega$ dynamo becomes possible, and this should continuously decrease the scatter in their $L_{\mathrm{X}} / L_{\mathrm{bol}}$ values. By the age of $\approx 30 \mathrm{Myr}$, nearly all stars have attained their final stellar structure, and thus the scatter in the activity levels has settled to the rather small dispersion as typical of (rapidly rotating) mainsequence stars. This scenario could be a qualitative explanation of the observed decrease in the scatter in the X-ray activity level of stars in the saturated rotation regime.

Acknowledgements. We would like to thank the LMU student Stefan Heigl for his help in collecting the literature data. We gratefully acknowledge support for this project from the Munich Cluster of Excellence: "Origin and Structure of the Universe".

\section{References}

Bouvier, J., Forestini, M., \& Allain, S. 1997, A\&A, 326, 1023

Briggs, K. R., Güdel, M., Telleschi, A., et al. 2007, A\&A, 468, 413

Broos, P. S., Feigelson, E. D., Townsley, L. K., et al. 2007, ApJS, 169, 353

Broos, P. S., Townsley, L. K., Feigelson, E. D., et al. 2010, ApJ, 714, 1582

Browning, M. K., \& Basri, G. 2007, Unsolved Problems in Stellar Physics, AIP

Conf. Proc., 948, 157

Chabrier, G., \& Küker, M. 2006, A\&A, 446, 1027

Cieza, L., \& Baliber, N. 2006, ApJ, 649, 862

Cieza, L., \& Baliber, N. 2007, ApJ, 671, 605

Cohen, R. E., Herbst, W., \& Williams, E. C. 2004, AJ, 127, 1602

Currie, T., \& Kenyon, S. J. 2009, AJ, 138, 703

Dib, S., Shadmehri, M., Padoan, P., et al. 2010, MNRAS, 405, 401
Dobler, W., Stix, M., \& Brandenburg, A. 2006, ApJ, 638, 336

Durney, B. R., De Young, D. S., \& Roxburgh, I. W. 1993, Sol. Phys., 145, 2070

Favata, F., \& Micela, G. 2003, Space Sci. Rev., 108, 577

Feigelson, E. D., \& Montmerle, T. 1999, ARA\&A, 37, 363

Getman, K. V., Flaccomio, E., Broos, P. S., et al. 2005, ApJS, 160, 319

Getman, K. V., Feigelson, E. D., Broos, P. S., Townsley, L. K., \& Garmire, G. P. 2010 ApJ, 708, 1760

Giampapa, M. S., Rosner, R., Kashyap, V., et al. 1996, ApJ, 463, 707

Güdel, M., Guinan, E. F., \& Skinner, S. L. 1997, ApJ, 483, 947

Güdel, M., Briggs, K. R., Arzner, K., et al. 2007, A\&A, 468, 353

Herbig, G. H. 1954, PASP, 66, 19

Herbig, G. H. 1998, ApJ, 497, 736

Herbst, W. 2008, in Handbook of Star Forming Regions, Vol. I, The Northern Sky ASP Monograph Publications, Vol. 4, ed. B. Reipurth, 372

Herbst, W., \& Mundt, R. 2005, ApJ, 633, 967

Herbst, W., Maley, J. A., \& Williams, E. C. 2000, AJ, 120, 349

Herbst, W., Eislöffel, J., Mundt, R., \& Scholz, A. 2007, in Protostars and Planets V, ed. B. Reipurth, D. Jewitt, \& K. Keil (Tucson: University of Arizona Press), 297

Iş1k, E., Schmitt, D., \& Schüssler, M. 2011, A\&A, 528, A135

James, D. J., Jardine, M. M., Jeffries, R. D., et al. 2000, MNRAS, 318, 1217

Jardine, M., \& Unruh, Y. C. 1999, A\&A, 346, 883

Jeffries, R. D., Jackson, R. J., Briggs, K. R., Evans, P. A., \& Pye, J. P. 2011, MNRAS, 411, 2099

Johns-Krull, C. M. 2007, ApJ, 664, 975

Kim, Y.-C., \& Demarque, P. 1996, ApJ, 457, 340

Kim, Y.-C., Fox, P. A., Demarque, P., \& Sofia, S. 1996, ApJ, 461, 499

Kızıloğlu, Ü., Kızıloğlu, N., \& Baykal, A. 2005, AJ, 130, 2766

Kraft, R. P., Burrows, D. N., \& Nousek, J. A. 1991, ApJ, 374, 344

Küker, M., \& Rüdiger, G. 1999, A\&A, 346, 922

Lada, E. A., \& Lada, C. J. 1995, AJ, 109, 1682

Lada, C. J., Muench, A. A., Luhman, K. L., et al. 2006, AJ, 131, 1574

Littlefair, S. P., Naylor, T., Burningham, B., \& Jeffries, R. D. 2005, MNRAS, 358,341

Luhman, K. L. 1999, ApJ, 525, 466

Luhman, K. L., Rieke, G. H., Lada, C. J., \& Lada, E. A. 1998, ApJ, 508, 347

Luhman, K. L., Stauffer, J. R., Muench, A. A., et al. 2003, ApJ, 593, 1093

Luhman, K. L., Lada, E. A., Muench, A. A., \& Elston, R. J. 2005, ApJ, 618, 810

Luhman, K. L., Allen, P. R., Espaillat, C., Hartmann, L., \& Calvet, N. 2010, ApJS, 186, 111

Mayne, N. J., Naylor, T., Littlefair, S. P., Saunders, E. S., \& Jeffries, R. D. 2007, MNRAS, 375, 1220

Maggio, A., Sciortino, S., Vaiana, G. S., et al. 1987, ApJ, 315, 687

Messina, S., Pizzolato, N., Guinan, E. F., \& Rodono, M. 2003, A\&A, 410, 671

Montesinos, B., Thomas, J. H., Ventura, P., \& Mazzitelli, I. 2001, MNRAS, 326,877

Muench, A. A., Lada, E. A., Lada, C. J., et al. 2003, AJ, 125, 2029 
F. Alexander and T. Preibisch: X-ray activity and rotation of the young stars in IC 348

Muench, A. A., Lada, C. J., Luhman, K. L., Muzerolle, J., \& Young, E. 2007, AJ, 134, 411

Nordhagen, S., Herbst, W., Rhode, K. L., \& Williams, E. C. 2006, AJ, 132, 1555

Ossendrijver, M. 2003, A\&AR, 11, 287

Pallavicini, R., Golub, L., Rosner, R., et al. 1981, ApJ, 248, 279

Pietarila Graham, J., Cameron, R., \& Schüssler, M. 2010, ApJ, 714, 1606

Pizzolato, N., Maggio, A., Micela, G., Sciortino, S., \& Ventura, P. 2003, A\&A, 397,147

Preibisch, T., \& Feigelson, E. D. 2005, ApJS, 160, 390

Preibisch, T., \& Zinnecker, H. 2001, AJ, 122, 866

Preibisch, T., \& Zinnecker, H. 2002, AJ, 123, 1613

Preibisch, T., \& Zinnecker, H. 2004, A\&A, 422, 1001

Preibisch, T., Zinnecker, H., \& Herbig, G. H. 1996, A\&A, 310, 456

Preibisch, T., Stanke, T., \& Zinnecker, H. 2003, A\&A, 409, 147

Preibisch, T., Kim, Y.-C., Favata, F., et al. 2005, ApJS, 160, 401

Prosser, C. F., Randich, S., Stauffer, J. R., Schmitt, J. H. M. M., \& Simon, T. 1996, AJ, 112, 1570
Randich, S. 1998, in Cool Stars, Stellar Systems, and the Sun, ASP Conf. Ser., 154,501

Randich, S. 2000, in Stellar Clusters and Associations: Convection, Rotation, and Dynamos, ed. R. Pallavicini, G. Micela, \& S. Sciortino, ASP Conf. Ser., 198, 401

Siess, L., Dufour, E., \& Forestini, M. 2000, A\&A, 358, 593

Stauffer, J. R., Balachandran, S. C., Krishnamurthi, A., et al. 1997a, ApJ, 475, 604

Stauffer, J. R., Hartmann, L. W., Prosser, C. F., et al. 1997b, ApJ, 479, 776

Stelzer, B., \& Neuhäuser, R. 2001, A\&A, 377, 538

Stelzer, B., Preibisch, T., Alexander, F., et al. 2012, A\&A, 537, A135

Telleschi, A., Güdel, M., Briggs, K., et al. 2005, ApJ, 622, 653

Townsley, L. K., Feigelson, E. D., Montmerle, T., et al. 2003, ApJ, 593, 874

Trujillo Bueno, J., Shchukina, N., \& Asensio Ramos, A. 2004, Nature, 430, 326

Vögler, A., \& Schüssler, M. 2007, A\&A, 465, L43

Weights, D. J., Lucas, P. W., Roche, P. F., Pinfield, D. J., \& Riddick, F. 2009, MNRAS, 392, 817 